PROCEEDINGS OF THE

AMERICAN MATHEMATICAL SOCIETY

Volume 139, Number 1, January 2011, Pages 309-317

S 0002-9939(2010)10657-3

Article electronically published on August 20, 2010

\title{
SURFACES IN FOUR-DIMENSIONAL HYPERKÄHLER MANIFOLDS WHOSE TWISTOR LIFTS ARE HARMONIC SECTIONS
}

\author{
KAZUYUKI HASEGAWA
}

(Communicated by Jon G. Wolfson)

\begin{abstract}
We determine surfaces of genus zero in self-dual Einstein manifolds whose twistor lifts are harmonic sections. We apply our main theorem to the case of four-dimensional hyperkähler manifolds. As a corollary, we prove that a surface of genus zero in four-dimensional Euclidean space is twistor holomorphic if its twistor lift is a harmonic section. In particular, if the mean curvature vector field is parallel with respect to the normal connection, then the surface is totally umbilic. Thus, our main theorem is a generalization of Hopf's theorem for a constant mean curvature surface of genus zero in threedimensional Euclidean space. Moreover, we can also see that a Lagrangian surface of genus zero in the complex Euclidean plane with conformal Maslov form is the Whitney sphere.
\end{abstract}

\section{INTRODUCTION}

For oriented surfaces in oriented four-dimensional Riemannian manifolds, the twistor lifts play an important role and have been studied by many researchers. For example, in [2, it is proved that any oriented surface admits a conformal, superminimal immersion into the four-dimensional sphere. The twistor space is endowed with an almost complex structure, which is integrable in case a base manifold is self-dual (see [1]). Surfaces with holomorphic twistor lifts are called twistor holomorphic surfaces (see [7]). On the other hand, the author studies surfaces whose twistor lifts are harmonic sections in [8] and [9]. If the ambient spaces are self-dual Einstein, the twistor lifts of twistor holomorphic surfaces are harmonic sections. Note that recently surfaces whose twistor lifts are harmonic sections have been studied from the viewpoint of the integrable systems in [3] and [11.

In this paper, we prove the following theorem.

Theorem. Let $\tilde{M}$ be a self-dual Einstein manifold with the scalar curvature $\tau$ and $M$ an oriented, connected, compact surface in $\tilde{M}$ with the Euler characteristic $\chi\left(T^{\perp} M\right)$ of the normal bundle $T^{\perp} M$. If the twistor lift of $M$ is a harmonic section and the genus of $M$ is zero, then we have

Received by the editors October 12, 2009 and, in revised form, April 1, 2010.

2000 Mathematics Subject Classification. Primary 53C42, 58E20.

This work is partially supported by the Grant-in-Aid for Young Scientists (B) No. 20740046, the Ministry of Education, Culture, Sports, Science and Technology, Japan.

(C)2010 American Mathematical Society Reverts to public domain 28 years from publication 
(1) $M$ is a non-superminimal minimal surface when $\chi\left(T^{\perp} M\right)>2-$ $(\tau / 24 \pi) \operatorname{Vol}(M)$

(2) $M$ is a superminimal surface when $\chi\left(T^{\perp} M\right)=2-(\tau / 24 \pi) \operatorname{Vol}(M)$,

(3) $M$ is a non-superminimal twistor holomorphic surface when $\chi\left(T^{\perp} M\right)<2-$ $(\tau / 24 \pi) \operatorname{Vol}(M)$.

Using this theorem, we have

Corollary. Let $\tilde{M}$ be a four-dimensional hyperkähler manifold and $M$ an oriented, connected, compact surface in $\tilde{M}$ with the Euler characteristic $\chi\left(T^{\perp} M\right)$ of the normal bundle $T^{\perp} M$. If the twistor lift of $M$ is a harmonic section and the genus of $M$ is zero, then we have

(1) $M$ is a non-superminimal minimal surface when $\chi\left(T^{\perp} M\right) \geq 4$,

(2) $M$ is a superminimal surface when $\chi\left(T^{\perp} M\right)=2$,

(3) $M$ is a non-superminimal twistor holomorphic surface when $\chi\left(T^{\perp} M\right) \leq 0$.

If certain conditions for the curvature tensors are satisfied and the twistor lift is a harmonic section, then the mean curvature vector field is a holomorphic section of the normal bundle with respect to the Koszul-Malgrange holomorphic structure (see [3], 8] and [9]). In general, holomorphic sections are not parallel. It is well-known that a compact constant mean curvature surface of genus zero immersed in $\mathbf{R}^{3}$ is totally umbilic, which was proved by Hopf in 1951. Many researchers generalize this theorem to various forms, for example, higher codimension cases (see 44). In these generalized results, the mean curvature vector field is often assumed to be parallel with respect to the normal connection. If the mean curvature vector field is parallel, then the surface is totally umbilic, using the corollary above (see [4] and 10, for example). Thus our main theorem is a generalization of Hopf's theorem to surfaces with holomorphic mean curvature vector fields. Moreover, we can also show that a Lagrangian surface of genus zero in the complex Euclidean plane with conformal Maslov form is the Whitney sphere (see [5]).

\section{Preliminaries}

Throughout this paper, all manifolds and maps are assumed to be smooth. Let $E$ be a vector bundle over a manifold $M$ and $E_{x}$ the fiber of $E$ over $x \in M$. We write $T P$ for the tangent bundle of a manifold $P$. For vector bundles $E, E^{\prime}$ over $M$, we denote the homomorphism bundle whose fiber is the space of linear mappings $E_{x}$ to $E_{x}^{\prime}$ by $\operatorname{Hom}\left(E, E^{\prime}\right)$, and set $\operatorname{End}(E):=\operatorname{Hom}(E, E)$. The space of all sections of a vector bundle $E$ is denoted by $\Gamma(E)$. Let $\varphi: N \rightarrow M$ be a smooth map and $E$ a vector bundle over $M$. The pull-back bundle of $E$ by $\varphi$ is denoted by $\varphi^{\#} E$.

Let $E$ be a Riemannian vector bundle with a fiber metric $g^{E}$ and a metric connection $\nabla^{E}$ over an $n$-dimensional Riemannian manifold $(M, g)$. Let $K^{E}$ : $T E \rightarrow E$ be the connection map with respect to $\nabla^{E}$. The canonical metric $G$ on $E$ is defined by

$$
G(\zeta, \zeta)=g\left(p_{*}(\zeta), p_{*}(\zeta)\right)+g^{E}\left(K^{E}(\zeta), K^{E}(\zeta)\right)
$$

for $\zeta \in T E$, where $p: E \rightarrow M$ is the bundle projection. We note that $p$ : $(E, G) \rightarrow(M, g)$ is a Riemannian submersion with totally geodesic fibers. Set $U E(=U(E)):=\left\{u \in E \mid g^{E}(u, u)=1\right\}$. The set of all sections $\xi \in \Gamma(E)$ such that $\xi(M) \subset U E$ is denoted by $\Gamma(U E)$. We assume that $M$ is compact. Let $\mathcal{E}$ be the energy functional defined on the space of all smooth maps from $M$ to $U E$. 
We say that $\xi \in \Gamma(U E)$ is a harmonic section (or vertically harmonic) if $\xi$ is a stationary point of $\left.\mathcal{E}\right|_{\Gamma(U E)}$. Obviously, if a section is a harmonic map in the usual sense, then it is a harmonic section. For harmonic sections, we refer to 15 .

Next we recall the twistor space and the twistor lift of a surface. Let $(\tilde{M}, \tilde{g})$ be an oriented four-dimensional Riemannian manifold. The Hodge star operator is denoted by $*$. Since $*^{2}=$ id for all 2 -forms, the bundle $\Lambda^{2}(\tilde{M})$ of all 2-forms on $\tilde{M}$ is decomposed into

$$
\Lambda^{2}(\tilde{M})=\Lambda_{+}^{2}(\tilde{M}) \oplus \Lambda_{-}^{2}(\tilde{M}),
$$

where $\Lambda_{ \pm}^{2}(\tilde{M})=\left\{\omega \in \Lambda^{2}(\tilde{M}) \mid * \omega= \pm \omega\right\}$. Using the metrics, we can identify $\Lambda_{-}^{2}(\tilde{M})$ with a vector subbundle $Q$ of $\operatorname{End}(T \tilde{M})$. We also write $\tilde{g}$ for the fiber metric of $Q$. A section $\phi \in \Gamma(U Q)$ satisfies $\phi^{2}=-I, \tilde{g}(\phi X, \phi Y)=\tilde{g}(X, Y)$ for all $X, Y \in T \tilde{M}$ and $-\Omega_{\phi} \wedge \Omega_{\phi}=d \mu$, where $\Omega_{\phi}$ is the fundamental form of $\phi$ and $d \mu$ is the volume form of $\tilde{M}$ compatible with the orientation. Note that $Q$ is a parallel subbundle in $\operatorname{End}(T \tilde{M})$ with respect to the connection which is induced by the Levi-Civita connection $\tilde{\nabla}$ of $\tilde{M}$. We use the same letter $\tilde{\nabla}$ for the induced connection. The twistor space $\mathcal{Z}$ over $\tilde{M}$ is the unit sphere bundle $U Q$ of $Q$. The bundle projection $p: \mathcal{Z} \rightarrow \tilde{M}$ and the connection $\tilde{\nabla}$ induce the decomposition

$$
T \mathcal{Z}=T^{h} \mathcal{Z} \oplus T^{v} \mathcal{Z}
$$

into the horizontal subbundle $T^{h} \mathcal{Z}$ and the vertical subbundle $T^{v} \mathcal{Z}$. On the twistor space $\mathcal{Z}$, almost complex structures $J_{i}^{\mathcal{Z}}(i=1,2)$ are defined by $J_{i}^{\mathcal{Z}}(X)=$ $\left(\phi\left(p_{*}(X)\right)\right)_{\phi}^{h}$ for all horizontal vectors $X$ at $\phi \in \mathcal{Z}$ and $J_{i}^{\mathcal{Z}}(V)=(-1)^{i-1} J^{v}(V)$ for all vertical vectors $V$, where $J^{v}$ is the canonical complex structure on each fiber $(\simeq$ the two-dimensional unit sphere). The almost complex structure $J_{1}^{\mathcal{Z}}$ on the twistor space is integrable if and only if $\tilde{M}$ is a self-dual manifold (see [1]). Note that $J_{2}^{\mathcal{Z}}$ is never integrable.

Let $f:(M, g) \rightarrow(\tilde{M}, \tilde{g})$ be an isometric immersion from an oriented surface $(M, g)$ into an oriented four-dimensional Riemannian manifold $(\tilde{M}, \tilde{g})$. The LeviCivita connection of $g$ is denoted by $\nabla$. Let $T^{\perp} M$ be the normal bundle of $f$ and $\nabla^{\perp}$ the normal connection of $T^{\perp} M$. We say that an orthonormal frame $\left(e_{1}, e_{2}, e_{3}, e_{4}\right)$ of $f^{\#}(T \tilde{M})$ is adapted if

(1) $e_{1}, e_{2}, e_{3}, e_{4}$ are compatible with the orientation of $\tilde{M}$,

(2) $e_{1}, e_{2}$ are compatible with the orientation of $M$,

(3) $e_{3}, e_{4}$ are normal to $M$.

Using an adapted frame, we define $J: T M \rightarrow T M$ by $J\left(e_{1}\right)=e_{2}$ and $J\left(e_{2}\right)=-e_{1}$, and $J^{\perp}: T^{\perp} M \rightarrow T^{\perp} M$ by $J^{\perp}\left(e_{3}\right)=-e_{4}$ and $J^{\perp}\left(e_{4}\right)=e_{3}$. It is evident that $\nabla J=0$ and $\nabla^{\perp} J^{\perp}=0$. We set

$$
\tilde{J}(X):=J(X) \text { and } \tilde{J}(\zeta):=J^{\perp}(\zeta)
$$

for $X \in T M$ and $\zeta \in T^{\perp} M$. Then $\tilde{J}$ is a section of $U\left(f^{\#} Q\right)\left(=f^{\#}(\mathcal{Z})\right)$ and $\tilde{J}$ is called the twistor lift of $M$. Hereafter, we often omit the symbol " $f$ " for the induced objects of the immersion $f$ if there is no confusion for the sake of simplicity. For example, we use the same letter $\tilde{\nabla}$ for the pull-back connection $f^{\#} \tilde{\nabla}$ by $f$. We say that $M$ is a superminimal surface if the twistor lift is a horizontal map, that is, $\tilde{\nabla} \tilde{J}=0$. If $\tilde{J}_{*} \circ J=J_{1}^{\mathcal{Z}} \circ \tilde{J}_{*}\left(\right.$ precisely, $\left(f_{\#} \circ \tilde{J}\right)_{*} \circ J=J_{1}^{\mathcal{Z}} \circ\left(f_{\#} \circ \tilde{J}\right)_{*}$, where $f_{\#}: U\left(f^{\#} Q\right) \rightarrow U Q$ is the bundle map), then $M$ is called a twistor holomorphic surface. See 7 for twistor holomorphic surfaces, for example. On the other hand, $M$ is minimal if and only if it follows that $\tilde{J}_{*} \circ J=J_{2}^{\mathcal{Z}} \circ \tilde{J}_{*}$ (see [6]). Let $\alpha$ (resp. 
$H$ ) be the second fundamental form (resp. the mean curvature vector field) of $M$. We define a $T^{\perp} M$-valued symmetric tensor $B$ by

$$
B(X, Y)=\alpha(X, J Y)-J^{\perp} \alpha(X, Y)+J^{\perp} \alpha(J X, J Y)+\alpha(J X, Y)
$$

for all $X, Y \in T M$. We see that $M$ is twistor holomorphic if and only if $B=0$ (see [7] and [8]). Note that a surface is superminimal if and only if $H=0$ and $B=0$ (see [7]). We define a function $\rho$ on $M$ by

$$
\rho=\tilde{g}\left(J^{\perp} \alpha\left(u_{1}, u_{1}\right)-\alpha\left(u_{1}, u_{2}\right), J^{\perp} \alpha\left(u_{2}, u_{2}\right)+\alpha\left(u_{1}, u_{2}\right)\right),
$$

where $\left(u_{1}, u_{2}\right)$ form an orthonormal frame on $M$ that is compatible with the orientation of $M$. If $M$ is superminimal, then $\rho=0$. We summarize the fundamental formulae for surfaces in self-dual Einstein manifolds with the scalar curvature $\tau$ which is needed in this paper. These are obtained by a straightforward calculation in 8 . Let $\mathcal{K}$ be the Gaussian curvature of $M$ and $\mathcal{K}^{\perp}$ the normal curvature of $T^{\perp} M$ defined by $\mathcal{K}^{\perp}=\tilde{g}\left(R_{e_{1}, e_{2}}^{\perp} e_{4}, e_{3}\right)$, where $R^{\perp}$ is the curvature form of the normal connection and $\left(e_{1}, \ldots, e_{4}\right)$ form an adapted frame. We have

$$
\begin{aligned}
& \mathcal{K}-\mathcal{K}^{\perp}=\frac{\tau}{12}+\rho, \\
& \|B\|^{2}=16\left(\frac{\tau}{12}+\|H\|^{2}-\mathcal{K}+\mathcal{K}^{\perp}\right), \\
& \|B\|^{2}=16\|H\|^{2}-16 \rho .
\end{aligned}
$$

Let $\chi(M)$ (resp. $\left.\chi\left(T^{\perp} M\right)\right)$ be the Euler characteristic of $M$ (resp. $T^{\perp} M$ ). In particular, by (2.2), if $M$ is compact, we have

$$
\frac{\tau}{24 \pi} \operatorname{Vol}(M)+\frac{1}{2 \pi} \int_{M}\|H\|^{2} d v_{g}-\chi(M)+\chi\left(T^{\perp} M\right) \geq 0 .
$$

The equality of (2.4) holds if and only if $M$ is twistor holomorphic.

\section{Proof of our main theorem}

In this section, we give the proof of our main theorem. To prove the main result, we use some fundamental results obtained in the author's papers [8] and $[9$. For $\beta \in \Gamma\left(\operatorname{Hom}\left(T M \otimes T M, T^{\perp} M\right)\right)$, we define $\nabla^{\prime} \beta$ by $\left(\nabla_{X}^{\prime} \beta\right)(Y, Z)=\nabla_{X}^{\perp} \beta(Y, Z)-$ $\beta\left(\nabla_{X} Y, Z\right)-\beta\left(Y, \nabla_{X} Z\right)$ for all $X, Y, Z \in \Gamma(T M)$. A $T^{\perp} M$-valued 1 -form $\delta \beta$ is defined by

$$
(\delta \beta)(X)=-\sum_{i=1}^{2}\left(\nabla_{u_{i}}^{\prime} \beta\right)\left(u_{i}, X\right)
$$

for all $X \in T M$, where $\left(u_{1}, u_{2}\right)$ is an orthonormal frame on $M$. When $\tilde{M}$ is a self-dual Einstein manifold, we obtain the following facts (see [9] and [16]).

Lemma 3.1. Let $M$ be a surface in a self-dual Einstein manifold. Then the following statements are mutually equivalent:

(1) The twistor lift $\tilde{J}$ is a harmonic section.

(2) $[\tilde{J}, \bar{\Delta} \tilde{\nabla} \tilde{J}]=0$, where $\bar{\Delta}^{\tilde{\nabla}}$ is the rough Laplacian of $\tilde{\nabla}$.

(3) The mean curvature vector $H$ satisfies $\nabla_{J}^{\perp} H=J^{\perp} \nabla_{X}^{\perp} H$ for all $X \in T M$.

(4) $\delta B=0$.

Lemma 3.2. Let $(M, g)$ be an oriented surface in a self-dual Einstein manifold $(\tilde{M}, \tilde{g})$ and $R^{Q}$ the curvature form of the pull-back connection on $f^{\#} Q$. Then we have $R_{X, Y}^{Q} \tilde{J}=0$ for all $X, Y \in T M$. 
The normal bundle $T^{\perp} M$ equips the Koszul-Malgrange holomorphic structure. Therefore, a section $\xi \in \Gamma\left(T^{\perp} M\right)$ is holomorphic relative to this structure if and only if $\nabla_{J X}^{\perp} \xi=J^{\perp} \nabla_{X}^{\perp} \xi$ for all $X \in T M$. With respect to the Koszul-Malgrange holomorphic structure, the twistor lift of a surface with holomorphic mean curvature vector field is a harmonic section when the ambient space is self-dual Einstein.

We note that the twistor lift depends on the orientation $M$ (or $\tilde{M}$ ). In particular, a surface is twistor holomorphic with respect to both orientations of $M$ if and only if the surface is totally umbilic. Similarly, when $\tilde{M}$ is self-dual Einstein, the twistor lifts are harmonic sections with respect to both orientations if and only if the mean curvature vector field is parallel.

We have the following theorem.

Theorem 3.3. Let $\tilde{M}$ be a self-dual Einstein manifold and $M$ an oriented, connected, compact surface in $\tilde{M}$. If the twistor lift of $M$ is a harmonic section and the genus of $M$ is zero, then we have

(1) $M$ is a non-superminimal minimal surface when $\chi\left(T^{\perp} M\right)>2-$ $(\tau / 24 \pi) \operatorname{Vol}(M)$,

(2) $M$ is a superminimal surface when $\chi\left(T^{\perp} M\right)=2-(\tau / 24 \pi) \operatorname{Vol}(M)$,

(3) $M$ is a non-superminimal twistor holomorphic surface when $\chi\left(T^{\perp} M\right)<$ $2-(\tau / 24 \pi) \operatorname{Vol}(M)$.

Proof. Let $(x, y)$ be an isothermal coordinate of $M$ and set $z=x+\sqrt{-1} y$. Bundles and some of other geometric objects, for example metrics, are extended $\mathbf{C}$-linearly with the same notation. We set $\partial_{z}:=(\partial / \partial z)$ and $\partial_{\bar{z}}:=(\partial / \partial \bar{z})$. By Lemma 3.1 , we see that $\tilde{J}$ is a harmonic section if and only if

$$
\left[\tilde{J}, \tilde{\nabla}_{\partial_{z}} \tilde{\nabla}_{\partial_{\bar{z}}} \tilde{J}+\tilde{\nabla}_{\partial_{\bar{z}}} \tilde{\nabla}_{\partial_{z}} \tilde{J}\right]=0 .
$$

On the other hand, by Lemma 3.2 the condition that $\tilde{M}$ be self-dual Einstein ensures that

$$
\left[\tilde{J}, \tilde{\nabla}_{\partial_{z}} \tilde{\nabla}_{\partial_{\bar{z}}} \tilde{J}-\tilde{\nabla}_{\partial_{\bar{z}}} \tilde{\nabla}_{\partial_{z}} \tilde{J}\right]=0 .
$$

Then, from (3.1) and (3.2), we conclude that $\tilde{J}$ is a harmonic section if and only if

$$
\left[\tilde{J}, \tilde{\nabla}_{\partial_{z}} \tilde{\nabla}_{\partial_{\bar{z}}} \tilde{J}\right]=\left[\tilde{J}, \tilde{\nabla}_{\partial_{\bar{z}}} \tilde{\nabla}_{\partial_{z}} \tilde{J}\right]=0 .
$$

Now we define a quadratic differential $q$ by $q=\operatorname{tr}\left(\tilde{\nabla}_{\partial_{z}} \tilde{J}\right)^{2} d z^{2}$. We have

$$
\partial_{\bar{z}} \operatorname{tr}\left(\tilde{\nabla}_{\partial_{z}} \tilde{J}\right)^{2}=2 \operatorname{tr}\left(\tilde{\nabla}_{\partial_{z}} \tilde{J}\right)\left(\tilde{\nabla}_{\partial_{\bar{z}}} \tilde{\nabla}_{\partial_{z}} \tilde{J}\right) .
$$

Since, by (3.31), $\tilde{\nabla}_{\partial_{\bar{z}}} \tilde{\nabla}_{\partial_{z}} \tilde{J}$ commutes with $\tilde{J}$ while $\tilde{\nabla}_{\partial_{z}} \tilde{J}$ and $\tilde{J}$ anti-commute, we have

$$
\begin{aligned}
\operatorname{tr}\left(\tilde{\nabla}_{\partial_{z}} \tilde{J}\right)\left(\tilde{\nabla}_{\partial_{\bar{z}}} \tilde{\nabla}_{\partial_{z}} \tilde{J}\right) & =\operatorname{tr}(-\tilde{J})\left(\tilde{\nabla}_{\partial_{z}} \tilde{J}\right)\left(\tilde{\nabla}_{\partial_{\bar{z}}} \tilde{\nabla}_{\partial_{z}} \tilde{J}\right) \tilde{J} \\
& =\operatorname{tr}\left(\tilde{\nabla}_{\partial_{z}} \tilde{J}\right)(\tilde{J} \tilde{J})\left(\tilde{\nabla}_{\partial_{\bar{z}}} \tilde{\nabla}_{\partial_{z}} \tilde{J}\right) \\
& =-\operatorname{tr}\left(\tilde{\nabla}_{\partial_{z}} \tilde{J}\right)\left(\tilde{\nabla}_{\partial_{\bar{z}}} \tilde{\nabla}_{\partial_{z}} \tilde{J}\right),
\end{aligned}
$$

and hence, $q$ is holomorphic. Thus $q$ vanishes on genus zero surfaces so that $\tilde{J}$ is holomorphic with respect to $J_{1}^{\mathcal{Z}}$ or $J_{2}^{\mathcal{Z}}$. We see that $M$ is minimal or twistor holomorphic.

Next we distinguish the possibilities via the Euler characteristic of $T^{\perp} M$. At first, we assume $\chi\left(T^{\perp} M\right)=2-(\tau / 24 \pi) \operatorname{Vol}(M)$. Then, by (2.1), we have

$$
\int_{M} \rho d v_{g}=0
$$


From (2.3), we obtain

$$
\int_{M}\|B\|^{2} d v_{g}=16 \int_{M}\|H\|^{2} d v_{g}
$$

Since $M$ is minimal or twistor holomorphic, $M$ is superminimal. When $\chi\left(T^{\perp} M\right)>$ $2-(\tau / 24 \pi) \operatorname{Vol}(M)$, we obtain

$$
\begin{aligned}
& \frac{\tau}{24 \pi} \operatorname{Vol}(M)+\frac{1}{2 \pi} \int_{M}\|H\|^{2} d v_{g}-\chi(M)+\chi\left(T^{\perp} M\right) \\
& \geq \frac{\tau}{24 \pi} \operatorname{Vol}(M)-2+\chi\left(T^{\perp} M\right) \\
& >0 .
\end{aligned}
$$

Then $M$ is not twistor holomorphic; that is, $M$ is a non-superminimal minimal surface. This is obvious for the case (3).

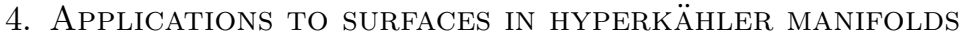

Let $\tilde{M}$ be a four-dimensional hyperkähler manifold and $I_{1}, I_{2}, I_{3}$ a hyperkähler structure on $\tilde{M}$. If an orientation of $\tilde{M}$ is given by

$$
-\sum_{i=1}^{3} \Omega_{I_{i}} \wedge \Omega_{I_{i}}
$$

then we have $I_{1}, I_{2}, I_{3} \in \Gamma(\mathcal{Z})$, where $\Omega_{I_{i}}$ is the fundamental form of $I_{i}(i=1,2,3)$. We note that $\tilde{M}$ is a self-dual Einstein (in fact, Ricci flat) manifold with respect to this orientation. We note that $\chi\left(T^{\perp} M\right)$ is an even integer if $\tilde{M}$ is a hyperkähler manifold. From Theorem 3.3, we have the following corollary immediately.

Corollary 4.1. Let $\tilde{M}$ be a four-dimensional hyperkähler manifold and $M$ an oriented, connected, compact surface in $\tilde{M}$. If the twistor lift of $M$ is a harmonic section and the genus of $M$ is zero, then we have

(1) $M$ is a non-superminimal minimal surface when $\chi\left(T^{\perp} M\right) \geq 4$,

(2) $M$ is a superminimal surface when $\chi\left(T^{\perp} M\right)=2$,

(3) $M$ is a non-superminimal twistor holomorphic surface when $\chi\left(T^{\perp} M\right) \leq 0$.

If $M$ is a non-superminimal twistor holomorphic surface in a hyperkähler manifold, then $\chi\left(T^{\perp} M\right) \leq 0$ (요). See [12] and [13] for minimal surfaces in hyperkähler manifolds, in particular, in $K 3$ surfaces with the hyperkähler metric, for example.

Using Corollary 4.1, we can see a quantization phenomenon for $\int_{M}\|H\|^{2} d v_{g}$.

Corollary 4.2. Let $\tilde{M}$ be a four-dimensional hyperkähler manifold and $M$ an oriented, connected, compact surface of genus zero in $\tilde{M}$. If the twistor lift is a harmonic section, then we have

$$
\frac{1}{4 \pi} \int_{M}\|H\|^{2} d v_{g} \in \mathbf{N} \cup\{0\} .
$$

Proof. From (2.4), we see that

$$
\frac{1}{2 \pi} \int_{M}\|H\|^{2} d v_{g}-2+\chi\left(T^{\perp} M\right) \geq 0 .
$$

Therefore, if

$$
\int_{M}\|H\|^{2} d v_{g}<4 \pi
$$


then we have

$$
\chi\left(T^{\perp} M\right) \geq 2-\frac{1}{2 \pi} \int_{M}\|H\|^{2} d v_{g}>0 .
$$

By Corollary 4.1, $M$ is minimal; otherwise $M$ is a non-superminimal twistor holomorphic surface. Hence, for this case, it follows that

$$
\frac{1}{4 \pi} \int_{M}\|H\|^{2} d v_{g}=1-\frac{1}{2} \chi\left(T^{\perp} M\right) \in \mathbf{N} .
$$

In the case where $\tilde{M}=\mathbf{R}^{4}$, we can obtain the following corollary immediately.

Corollary 4.3. Let $M$ be an oriented, compact, connected surface of genus zero in $\mathbf{R}^{4}$. If the twistor lift of $M$ is a harmonic section, then $M$ is a non-superminimal twistor holomorphic surface.

Proof. Since $\mathbf{R}^{4}$ does not admit any compact minimal surface, $M$ is a nonsuperminimal twistor holomorphic surface by Corollary 4.1 .

Since the property that $M$ is twistor holomorphic is invariant under conformal changes of the metric on $\tilde{M}$, we can obtain many twistor holomorphic surfaces of genus zero in $\mathbf{R}^{4}$ from superminimal surfaces in the four-dimensional sphere.

In general, the holomorphic mean curvature vector field is not parallel. In the case where the mean curvature vector is parallel, using Corollary 4.3, we have the following corollary (see [4] and [10, for example).

Corollary 4.4. Let $M$ be an oriented, connected, compact surface of genus zero in $\mathbf{R}^{4}$. If the mean curvature vector field is parallel with respect to the normal connection, then $M$ is totally umbilic.

Proof. Since the mean curvature vector is parallel, the twistor lift is a harmonic section. Moreover, with respect to the opposite orientation of $M$, its twistor lift is also a harmonic section. By Corollary 4.3, $M$ is twistor holomorphic with respect to both orientations, and hence, $M$ is totally umbilic.

It is easy to obtain Hopf's theorem for a constant mean curvature surface of genus zero in $\mathbf{R}^{3}$ by using Corollary 4.4. In fact, by considering the totally geodesic immersion from $\mathbf{R}^{3}$ to $\mathbf{R}^{4}$, the constant mean curvature surface can be seen as an immersed surface in $\mathbf{R}^{4}$ with parallel mean curvature vector field. Thus our main theorem is a generalization of Hopf's theorem.

Here we recall the Grassmann manifold and the Gauss map. Let $G_{o}^{4,2}$ be the Grassmann manifold of all oriented 2-planes in $\mathbf{R}^{4}$. The Grassmann manifold $G_{o}^{4,2}$ is isomorphic to $S_{+}^{2}(1) \times S_{-}^{2}(1)$, where $S_{ \pm}^{2}(1)$ are the unit spheres in the 3 -dimensional vector spaces of all self-dual and anti-self-dual two-forms, respectively. Let $p_{ \pm}$: $G_{o}^{4,2}\left(\cong S_{+}^{2}(1) \times S_{-}^{2}(1)\right) \rightarrow S_{ \pm}^{2}(1)$ be the projection onto each factor of the product of the spheres. On the other hand, the twistor space $\mathcal{Z}$ of $\mathbf{R}^{4}$ is $\mathbf{R}^{4} \times S^{2}(1)$. The projection from $\mathcal{Z}$ onto $S^{2}(1)$ is denoted by $\hat{p}$. The map $f_{\#} \circ \tilde{J}$ can be identified with the map $M \ni x \mapsto \omega_{1} \wedge \omega_{2}-\omega_{3} \wedge \omega_{4}$, where $\left(\omega_{1}, \ldots, \omega_{4}\right)$ is the dual frame of an adapted frame $\left(e_{1}, \ldots, e_{4}\right)$ at each point $x \in M$. Then we have

$$
\left(\hat{p} \circ f_{\#} \circ \tilde{J}=\right) \hat{p} \circ \tilde{J}=p_{-} \circ \varphi,
$$

where $\varphi: M \rightarrow G_{o}^{4,2}$ is the Gauss map of $M$ in $\mathbf{R}^{4}$. Therefore, if the twistor lift of $M$ in $\mathbf{R}^{4}$ is a harmonic section, then the half part of the Gauss map is harmonic. On 
the other hand, surfaces in Euclidean space whose (whole) Gauss map is harmonic have parallel mean curvature vector fields (see [14).

Finally, we give an application to a Lagrangian surface in $\mathbf{C}^{2}\left(\cong \mathbf{R}^{4}\right)$. Let $(\tilde{M}, \tilde{g}, \phi)$ be a four-dimensional Kähler manifold with the complex structure $\phi$ and denote the Kähler form of $M$ by $\Omega$. If a surface $M$ in $\tilde{M}$ is Lagrangian, that is, $\phi(T M)=T^{\perp} M$, then we define the Maslov form $\omega$ on $M$ by $\omega(X)=$ $(1 / \pi) \Omega(X, \phi H)$ for all $X \in \Gamma(T M)$. For a Lagrangian surface $M$, the Maslov form on $M$ is said to be conformal if the tangent vector $\phi H$ is a conformal vector field (see [5]). Define an immersion $w: S^{2}(1) \rightarrow \mathbf{C}^{2}$ by

$$
w(x, y, z)=\frac{1}{1+z^{2}}(x(1+\sqrt{-1} z), y(1+\sqrt{-1} z)) .
$$

The immersion $w$ is called the Whitney immersion or the Whitney sphere, which is a Lagrangian immersion. Since the Whitney sphere is twistor holomorphic, it is a Willmore surface; that is, it is a stationary point for the Willmore functional. By using Corollary 4.3, we have the following fact, which is proved in [5].

Corollary 4.5. Let $M$ be an oriented, connected, compact, Lagrangian surface of genus zero in $\mathbf{C}^{2}$. If the Maslov form on $M$ is conformal, then $M$ is congruent to the Whitney sphere.

Proof. If the Maslov form of $M$ is conformal, then $p_{-} \circ \varphi$ is harmonic (see [5]). Therefore $\hat{p} \circ \tilde{J}$ is also a harmonic map, and hence, the twistor lift of $M$ is a harmonic section (see also [8]). By Corollary 4.3, we see that $M$ is twistor holomorphic. Since the Whitney sphere is the only Lagrangian Willmore surface of genus zero in $\mathbf{C}^{2}$, $M$ is the Whitney sphere.

\section{ACKNOWLEDGMENTS}

This paper was prepared during the author's visit in August 2008 to Technische Universität Berlin as a guest researcher. The author would like to thank Technische Universität Berlin for its hospitality and to express his sincere gratitude to Professor Udo Simon for valuable discussions and kindness. He would like to thank Professor Naoto Abe for his constant encouragement. He would also like to thank the referees for their comments and suggestions to improve this paper.

\section{REFERENCES}

[1] M. F. Atiyah, N. J. Hitchin and I. M. Singer, Self-duality in four-dimensional Riemannian geometry, Proc. R. Soc. London Ser. A 362 (1978) 425-461. MR.506229 (80d:53023)

[2] R. L. Bryant, Conformal and minimal immersions of compact surfaces into the 4-sphere, J. Diff. Geom. 17 (1982) 455-473. MR679067 (84a:53062)

[3] F. Burstall and I. Khemar, Twistors, 4-symmetric spaces and integrable systems, Math. Ann. 344 (2009) 451-461. MR2495778

[4] B. Y. Chen, Geometry of submanifolds, Pure and Applied Mathematics, No. 22, Marcel Dekker, Inc., New York, 1973. MR0353212 (50:5697)

[5] I. Castro and F. Urbano, Lagrangian surfaces in the complex Euclidean plane with conformal Maslov form, Tohoku Math. J. (2) 45 (1993) 565-582. MR1245723 (94j:53064)

[6] J. Eells and S. Salamon, Twistorial construction of harmonic maps of surfaces into fourmanifolds, Ann. Scuola Norm. Sup. Pisa Cl. Sci. (4) 12 (1985), no. 4, 589-640 (1986). MR 848842 (87i:58042)

[7] T. Friedrich, On surfaces in four-spaces, Ann. Global Anal. Geom. 2 (1984) 275-287. MR7777909 (86h:53061) 
[8] K. Hasegawa, On surfaces whose twistor lifts are harmonic sections, J. Geom. Phys. 57 (2007) 1549-1566. MR 2310605 (2009b:53074)

[9] K. Hasegawa, Stability of twistor lifts for surfaces in four-dimensional manifolds as harmonic sections, J. Geom. Phys. 59 (2009) 1326-1338. MR2541823

[10] S. Hirakawa, Constant Gaussian curvature surfaces with parallel mean curvature vector in two-dimensional complex space forms, Geom. Dedicata 118 (2006), 229-244. MR2239458 (2007c:53073)

[11] I. Khemar, Geometric interpretation of second elliptic integrable systems, Differ. Geom. Appl. 28 (2010) 40-64. MR2579382

[12] M. Micallef and J. Wolfson, The second variation of area of minimal surfaces in four-manifolds, Math. Ann. 295 (1993) 245-267. MR1202392 (94c:58035)

[13] M. Micallef and J. Wolfson, Area minimizers in a $K 3$ surface and holomorphicity, Geom. Funct. Anal. 16 (2006) 437-452. MR2231469 (2007b:53125)

[14] E. Ruh and J. Vilms, The tension field of the Gauss map, Trans. Amer. Math. Soc. 149 (1970) 569-573. MR0259768 (41:4400)

[15] C. M. Wood, The energy of Hopf vector fields, Manuscripta Math. 101 (2000) 71-88. MR1737225 (2001c:53090)

[16] C. M. Wood, Harmonic sections of homogeneous fibre bundles, Differential Geom. Appl. 19 (2003) 193-210. MR2002659 (2004f:53078)

Faculty of Teacher Education, Institute of Human and Social Sciences, Kanazawa University, KaKUMA-MACHI, KANAZAWA, IshikAWA, 920-1192, JAPAN

E-mail address: kazuhase@staff.kanazawa-u.ac.jp 